\title{
KERATOPLASTY*
}

\section{A METHOD USING TRACK SUTURES AND THE OPERATING MICROSCOPE}

\author{
BY
}

NORMAN SANDERS

Department of Ophthalmology, University of Miami School of Medicine, Bascom Palmer Eye Institute, Miami, Florida

THE purpose of this paper is to demonstrate an improved method of suturing corneal grafts. Technically, the first few sutures are especially difficult and frequently the graft suffers undesirable damage. This becomes quite obvious when one sees in the literature the multitude of devices available for fixing the donor graft. With the corneal transplant technique described below, trauma to the tissues is minimized. Although not complicated, the method to be described requires skill with the operating microscope (Fig. 1), which once mastered becomes an indispensable tool for the operator. In addition, pre-placed tracks, somewhat similar to the track suture described by Verhoeff (1927) in cataract surgery, are used.

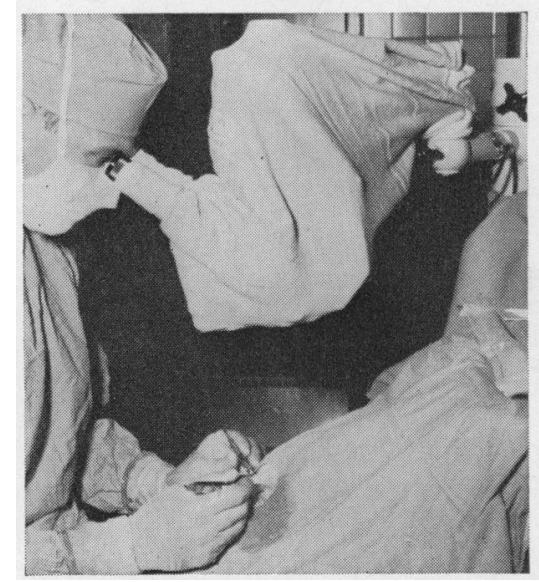

FIG. 1.-Operator shown with microscope which is covered with a sterile sleeve.

\section{Donor Eyes}

Whenever possible, eyes no older than 24 hours are used for penetrating grafts Stocker (1953) and Stocker, Matton, Eiring, Georgiade, and Georgiade (1960). They are stored at $4^{\circ} \mathrm{C}$. in a sterile jar which is moistened by pouring into it approximately $15 \mathrm{ml}$. of a suitable antibiotic solution. Eyes preserved in this way for 4 to 5 days may be used for lamellar transplants, but an accidental perforation into the anterior chamber or an unexpected finding at surgery may necessitate the change to a penetrating graft and for this reason, it is best to delay surgery until fresh material is available. 


\section{Technique}

The trephine is set at 0.2 to $0.3 \mathrm{~mm}$. to make an incision approximately one-half the way through the donor cornea (Fig. 2).

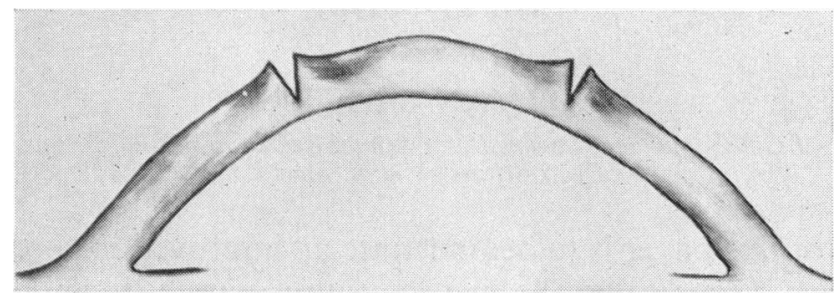

Fig. 2.-Partial incision into donor cornea.

The assistant cradles the donor eye between his index and middle fingers, palm down. The operator exerts gentle pressure with a 4.0 or 5.0 silk suture in the $90^{\circ}$ and $180^{\circ}$ meridian, thus marking four equidistant spots on the graft edge (Fig. 3). $\dagger$

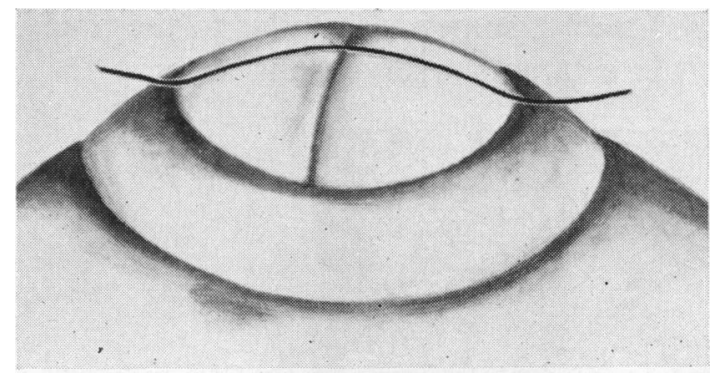

FIG. 3.-Marking graft for placement of first four tracks. In one meridian, the suture can be seen marking the cornea. The other meridian demonstrates the indentation it leaves (technique of Dr. Max Fine).

Next a sharp Riedel or Grieshaber needle is passed through the incision at the sites previously marked (Figs 4 and 5, opposite). With each track the eye of the needle is filled by touching it with a cotton tip applicator saturated with methylene blue. Additional tracks are placed in each quadrant as desired, the total number equalling the number of sutures to be inserted.

Sixteen sutures are required in a $7 \mathrm{~mm}$. graft. Since 8.0 silk is used, this large number of sutures presents no problem; on the contrary, wound closure is excellent and overriding of the wound edges unusual.

The advantages thus far gained are these: since the corneal button is firmly attached to the globe there is no twisting or turning of the graft. The globe is rotated after each track is placed, so that the operator is working in the most comfortable position. Consequently, perfectly radial tracks close to the wound edge are possible. Since all this is being done under microscopic control, each track is placed at the proper depth. 


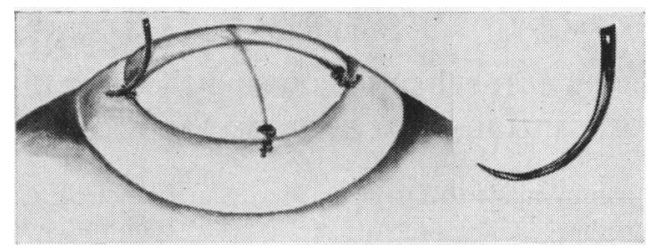

Fig. 4.-Needle with methylene blue passed through trephined edge.

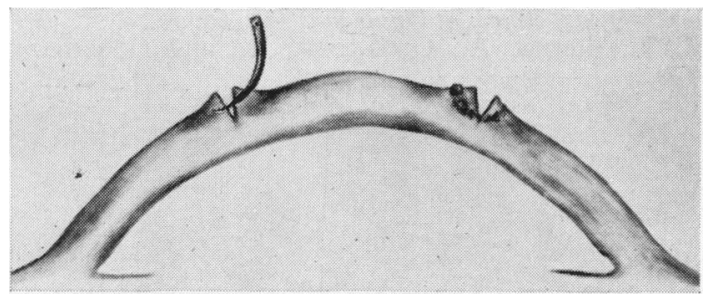

FIG. 5.-Cross-section of Fig. 4.

The next step is to put the same trephine in the groove, and an attempt is made to remove a clean disc with only three or four twists of the trephine (Fig. 6). Persistence of this manoeuvre can damage the endothelium by excessive contact with the lens-iris diaphragm. When necessary, the incision is completed with scissors.

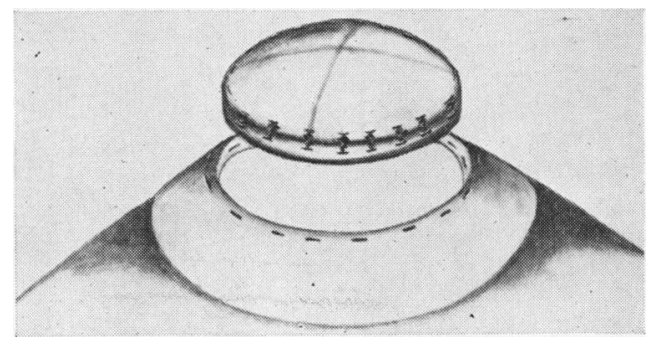

FIG. 6.-Graft with methylene blue tracks all
coming out at a uniform depth.

The recipient eye is now ready for trephining. Fixation is achieved by placing forceps on the medial and lateral recti, taking care neither to depress nor to elevate the globe. At the first sign of aqueous, the trephine is removed. The pupil is miotic from pre-operative pilocarpine, but extreme care must be taken to avoid damage to the lens and iris. Again, the incision is completed with scissors.

The donor button is now placed on the open eye and the sutures are put in by slipping the needle, now threaded with 8.0 silk, into the tracks and out through the recipient cornea. The operating microscope is invaluable for picking up the entry into the tracks and for judging the depth of the needle bite in the recipient cornea.

The end of the procedure should find the graft and recipient edges in perfect apposition and water-tight. In an aphakic graft, air is injected into the anterior chamber as is generally recommended to keep the vitreous away from the back of the cornea. 


\section{Summary}

A method of placing corneal sutures, using the operating microscope and track sutures, in corneal transplant surgery, has been described.

Acknowledgement is hereby made to Dr. Max Fine, San Francisco, California. Many of his teachings have been expressed in this paper, but have not been individually listed. I am also indebted to Dr. Bernd Silver and Miss Barbara Tarr for the illustrations.

\section{REFERENCES}

STOCKER, F. W. (1953). Trans. Amer. ophthal. Soc., 51, 669.

, Matton, M. T., Eiring, A., Georgiade, R., and Georgiade, N. (1960). Amer. J. Ophthal., 49, 729.

Verhoeff, F. H. (1927). Trans. Amer. ophthal. Soc., 25, 48. 